\title{
Estimation of the infectious reservoir of Plasmodium falciparum in natural vector populations based on oocyst size
}

H. Haji ${ }^{1,}{ }^{2}$, T. Smith ${ }^{3}$, J. Th. Meuwissen ${ }^{4}$, R. Sauerwein ${ }^{4}$ and J. D. Charlwood ${ }^{1}{ }^{1}$ Ifakara Centre, P.O. Box 53 Ifakara, Tanzania; ${ }^{2}$ Ministry of Health, P.O. Box 236, Zanzibar, Tanzania; ${ }^{3}$ Swiss Tropical Institute, P.O. Box, CH-4002 Basel, Switzerland; ${ }^{4}$ Department of Medical Microbiology, University of Nijmegen, P.O. Box 9101, 6500 HB Nijmegen, The Netherlands

\begin{abstract}
A method for determining the infectious reservoir of malaria $(K)$ and vector survival rate $(P)$ by measuring oocyst size and discriminating between the most recent and other infections is described. In the laboratory the mean diameter of $3 \mathrm{~d}$ oocysts in Anopheles gambiae, kept at $26^{\circ} \mathrm{C}$, was $11.5 \mu \mathrm{m}$ and the mean diameter at day 5 was $24.5 \mu \mathrm{m}$. Oocyst sizes in wild caught mosquitoes from southern Tanzania, that had fed on the occupants of bed nets with holes in the sides, were more variable. $2060 \mathrm{~A}$. gambiae s.l. and $1982 \mathrm{~A}$. funestus were examined for oocysts $3 \mathrm{~d}$ after feeding; 796 and 654 oocysts from the 153 and 170 infected females, respectively, were measured. Because of misclassification errors, the use of a simple cut-off model, in which all oocysts less than $17.5 \mu \mathrm{m}$ in diameter were considered to have arisen from the most recent feed, was thought to overestimate $K$ and underestimate $P$. A statistical model which allows for overlap in the oocyst size distributions is described. Estimates of the infectious reservoir derived from this model were $2 \cdot 8 \%$ for A. gambiae s.l. and $4 \cdot 2 \%$ for $A$. funestus, and the estimated survival rates per gonotrophic cycle were $65 \cdot 5 \%$ and $52.9 \%$, respectively. The utility of measuring oocyst size in naturally infected mosquitoes is discussed.
\end{abstract}

Keywords: malaria, Plasmodium falciparum, reservoir in vector, Anopheles gambiae s.l., Anopheles funestus, oocyst size

\section{Introduction}

Epidemiological studies of transmission of malaria from humans to mosquitoes have been carried out both to assess the infectious reservoir in humans for mosquitoes (MUIRHEAD-THOMSON \& MERCIER, 1952; DRAPER, 1953; MUIRHEAD-THOMSON, 1954, 1957; GRAVES et al., 1988, 1990; GITHEKO et al., 1992; THUIKAM et al., 1993; Charlwood et al., 1995; J. D. Charlwood et al., in preparation) and to identify factors which modify the infectiousness of individual hosts (HAJI et al., in press).

Infectivity can be studied in feeding experiments using local vectors, raised from wild-caught larvae or pupae (MUIRHEAD-THOMAS \& MERCIER, 1952; MUIRHEADTHOMSON, 1954, 1957; GRAVES et al., 1990) or colony-adapted mosquitoes (GITHEKo et al., 1992; THUIKAM et al., 1993). In either case mosquitoes that have fed on known hosts are generally kept alive for 5-7 d, long enough for oocyst infections to be apparent by microscopical examination of their midguts. The proportion with infections $(D$; the delayed oocyst rate) then gives an estimate of infectivity. On the other hand, laboratoryadapted insects may differ from the local population in their susceptibility to infection.

In contrast, the use of bed nets with holes in them to catch mosquitoes does not require the establishment of mosquito colonies, estimates infectivity using local vectors, an'd allows the mosquitoes to feed naturally. Delayed oocyst rates from bed nets with holes have been used to estimate the infectious reservoir $(K)$ (CHARLWOOD et al., in press), but when the study requires the identification of the source of the mosquito infections it is less than ideal because oocysts may be derived from more than one feed (HAJI et al., in press); $1-K / D$ then gives an estimate of the proportion of the detected infections that were acquired before the last feed. Estimates of $K$ derived from parous rates together with values of $D$ from collections using bed nets with holes in Namawala (J. D. Charlwood et al., in preparation) indicated that about $60 \%$ of oocysts may have been derived from previous fecds. Using mark-recapture methods, BABIKER et al. (1995) estimated that about onequarter of the infected Anopheles gambiae s.l. and oneeighth of the infected $A$. funestus caught in bed nets with holes had obtained their infections elsewhere.

In this study we report on a modification of the bed nets with holes technique that seeks to avoid the poten-

Address for correspondence: Tom Smith, Department of Public Health and Epidemiology, Swiss Tropical Institute, P.O. Box, CH-4002 Basel, Switzerland; fax +41612717951 . tial contamination of recent oocysts with pre-existing infections. This makes it possible both to estimate $K$ directly and to study the infectiousness of individual hosts. The method relies on the detection of the oocysts shortly after the blood meal disappears from the stomach of the mosquito. At this stage the oocysts are much smaller than those aged $5 \mathrm{~d}$ or more, and can be distinguished by their size from previously acquired infections (SHUTE \& MARYON, 1952; MUIRHEAD-THOMSON, 1957).

\section{Materials and methods \\ Experiments using bed nets with holes}

From February to December 1994 mosquitoes were collected from volunteers in the village of Kisegese, southern Tanzania (described by HAJI et al., in press) by the use of traps consisting of a cone-shaped bed net with $2-4$ juxtaposed holes about $6 \mathrm{~cm}$ in diameter halfway up the sides.

Mosquitoes were collected each morning into paper cups and transported to the field laboratory where they were kept until digestion of the blood meal had been completed (which took 2-3 d, depending on the temperature).

Midguts were dissected in $2 \%$ mebromine solution and transferred into $4 \%$ phosphate-buffered formaldehyde in $2 \mathrm{~mL}$ plastic vials for preservation. Up to 40 midguts were stored per vial. They remained in good condition for more than a year, which facilitated batch processing. Preserved midguts were mounted in $4 \%$ phosphate-buffered formaldehyde, covered with a coverglass, and examined at $\times 40$ with a compound microscope. The presence of very small oocysts was sometimes confirmed using an oil immersion objective. Oocysts from preserved midguts were measured to an accuracy of $0.3 \mu \mathrm{m}$ using an ocular graticule.

\section{Laboratory determination of oocyst growth rate}

The rate of development of oocysts was determined using a colony of $A$. gambiae s.s. in the laboratory, fed on cultured gametocytes of the Pf54 strain of Plasmodium falciparum. After feeding, mosquitoes were kept at $26^{\circ} \mathrm{C}$ for 3-5 d when they were processed as described above.

\section{Data analysis}

Let $K$ be the probability that an anthropophagic mosquito acquired an infection at the latest feed, $R$ the probability that it was already infected (equivalent to the immediate oocyst rate in mosquitoes coming to feed), and $D$ the overall probability that a mosquito is infected (the 
delayed oocyst rate). The ratio $R / \mathrm{D}$ can be used as an estimate of $P$, the survival per gonotrophic cycle (SERVICE, 1993; J. D. Charlwood et al., in preparation). D is estimated directly as the overall infection prevalence, whilst the estimation of $K$ and $R$ is more problematical.

In order to estimate $K$, it was necessary to estimate the proportion of mosquitoes infected at the most recent feed, and therefore to determine the proportion of oocysts that were derived from that feed. Let $x$ denote the size of an oocyst, $\theta(x)$ the distribution of oocyst sizes when the infection was acquired at the most recent feed and $\phi(x)$ the distribution of oocyst sizes from previous infections. The overall distribution of oocyst sizes is then:

$$
f(x) \sim(1-\lambda) \theta(x)+\lambda \phi(x)
$$

where the mixing proportion, $\lambda$, is the proportion of oocysts that resulted from previous feeds. Note that this is different from the distribution of oocyst sizes in mosquitoes with infections derived from both the latest and previous feed(s), which has a mixing proportion $\beta$ and is consequently

$$
f^{\prime}(x) \sim(1-\beta) \theta(x)+\beta \phi(x)
$$

(i) The simplest approach was to consider all oocysts with diameters below a given cut-off size as those from the most recent feed, and all others to be from earlier feeds: This allowed classification of individual mosquitoes and therefore provided direct estimates of $\lambda, D$ and $R$. It did not allow for overlap between the 2 distributions, $\theta(x)$ and $\phi(x)$, and therefore presumably resulted in some misclassification of mosquitoes.

(ii) The second method estimated the extent of overlap between the distributions. The distribution of oocysts in laboratory mosquitoes kept for $3 \mathrm{~d}$ can be considered as an estimate of $\theta(x)$. By comparing the observed $f(x)$ with this distribution, estimates of $\lambda$ and $\phi(x)$ were obtained, using the method of VOUNATSOU et al. (in press).

Using these estimates of $D, \lambda, \theta(x)$ and $\phi(x)$, estimates of $K$ were obtained by maximum likelihood (see the Appendix). The estimation procedure assumed that all mosquitoes were equally susceptible, and that the probabilities of acquiring an infection at successive feeds wcrc independent. It follows that

$$
D=R+K-R K
$$

and hence $R=(D-K) /(1-K)$

Considering all infections, the expected number of oocysts from the most recent feed per infected mosquito is $N(1-\beta) K / D$ and from previous feed(s) it is $N \beta R / D$; the ratio of the 2 values is $\beta R /((1-\beta) K)$. Therefore:

$$
\lambda /(1-\lambda)=\beta R /((1-\beta) K)
$$

Rearranging this gives

$$
\beta=\lambda K /(R+\lambda(K-R))
$$

and estimates of $\beta$ and $R$ can therefore be obtained from the maximum likelihood estimates of $K$.

\section{Results}

The Figure shows the distribution of oocyst sizes 3 and $5 \mathrm{~d}$ after feeding in the laboratory, and the distribution of oocyst sizes in field-caught mosquitoes $3 \mathrm{~d}$ after feeding. The mean diameter of 29 oocysts from 13 mosquiloes examined $3 \mathrm{~d}$ after an infectious blood meal in the laboratory was $11.5 \mu \mathrm{m}(\mathrm{SD}=2.4)$, whilst after $5 \mathrm{~d}$ the mean diameter of 78 oocysts from 15 mosquitoes examined was $24.5 \mu \mathrm{m}(\mathrm{SD}=4.5)$ Geometric mean numbers of oocyst per gut were 2.0 for the $3 \mathrm{~d}$ oocysts and 2.9 for those examined at $5 \mathrm{~d}$. The difference in oocyst numbers was largely due to 2 midguts in the latter category that contained 16 and 10 oocysts respectively.
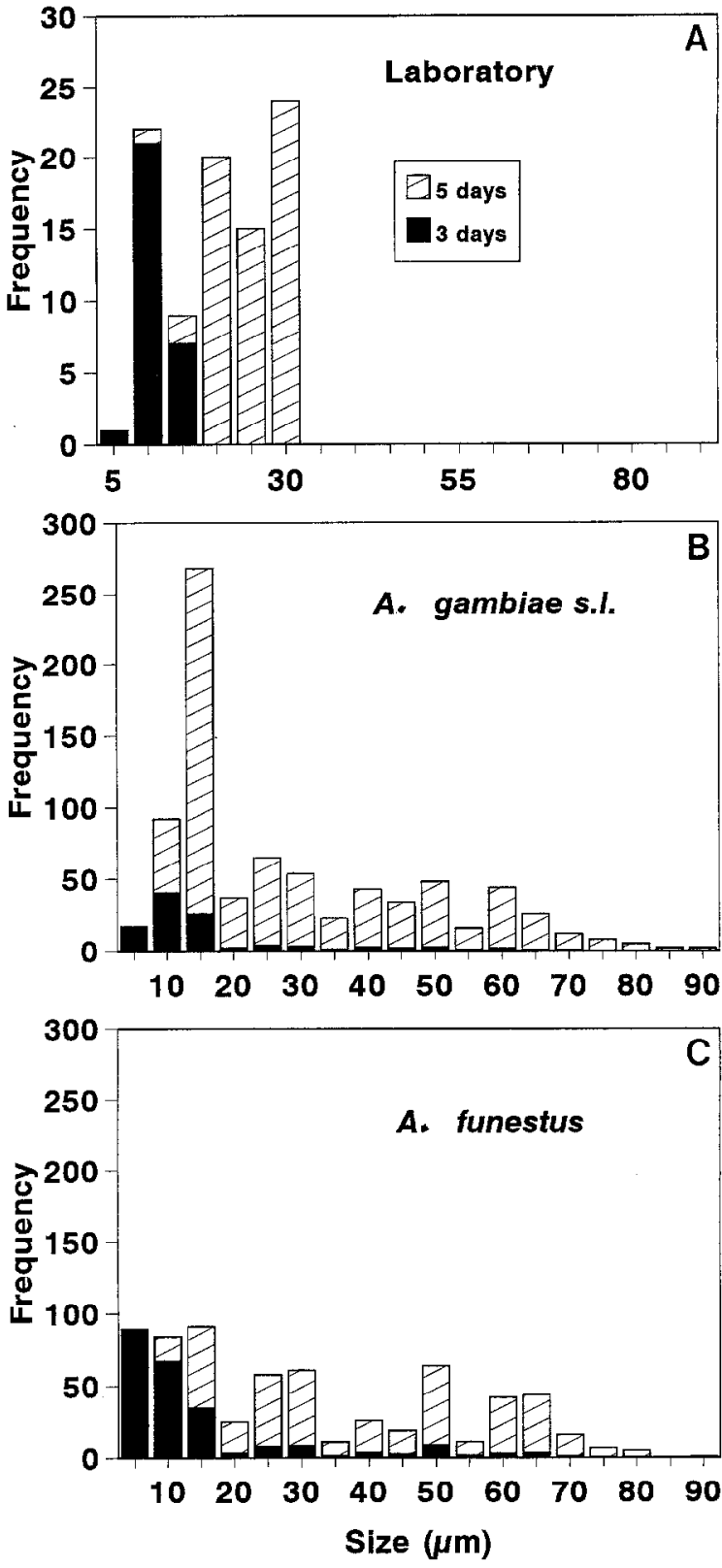

Figure. Distributions of oocyst sizes in laboratory-reared $A$. gambiae s.s. (A) and field-caught $A$. gambiae s.l. (B) and $A$. funestus (C). In panels B and C, solid columns indicate oocysts estimated to result from the latest feed and hatched columns indicate oocysts estimated to result from previous feeds.

153 A. gambiae s.l. (from a total of 1987 examined) and $170 \mathrm{~A}$. funestus (from a total of 1982 examined) from the bed nets with holes catches contained oocysts (of all sizes) 2-3 d after feeding. The consequent values of $D$ are given in the Table. The geometric mean number of oocysts per gut was 3.03 (total of 796 oocysts) in $A$. gambiae s.l. and 2.55 (654 oocysts) in $A$. funestus, twice that found in a nearby village (1.55 for A. gambiae s.l. and 0.93 for $A$. funestus) (BILLINGSLEY et al., 1994).

In field-caught mosquitoes the distribution of size ranges was not as clear cut as the distribution in mosquitoes that had fed in the laboratory. This suggests that oocysts from more than one feed were present.

After inspection of only the laboratory data, the cutoff value to discriminate $3 \mathrm{~d}$ oocysts from older ones was chosen as $17.5 \mu \mathrm{m}$. Using this approach, $293(42.9 \%)$ of the oocysts in field-caught $A$. funestus and $406(49 \cdot 2 \%)$ of those in $A$. gambiae s.l. were considered to be derived 
Table. Estimates of percentages of mosquitoes with infections of $P$. falciparum resulting from different feeds

\begin{tabular}{|c|c|c|c|c|}
\hline & \multicolumn{2}{|c|}{ A. funestus } & \multicolumn{2}{|c|}{ A. gambiae s.l. } \\
\hline & Cut-off & Decomposition & Cut-off & Decomposition \\
\hline All infections $(D)$ & $8 \cdot 6$ & $8 \cdot 6$ & $7 \cdot 7$ & $7 \cdot 7$ \\
\hline $\begin{array}{l}\text { Infective feed } \\
\text { Last feed only } \\
\text { Previous feed(s) only }\end{array}$ & $\begin{array}{l}4 \cdot 2 \\
3 \cdot 6\end{array}$ & $\begin{array}{l}4 \cdot 1 \\
4 \cdot 4\end{array}$ & $\begin{array}{l}2.9 \\
3.8\end{array}$ & $\begin{array}{l}2 \cdot 7 \\
4 \cdot 9\end{array}$ \\
\hline $\begin{array}{l}\text { Mixed infections } \\
\text { Estimated } \\
\text { Expected }\end{array}$ & $\begin{array}{l}0.71 \\
0.21\end{array}$ & $\overline{0} \cdot \overline{19}$ & $\begin{array}{l}0.65 \\
0.16\end{array}$ & $\overline{0} \cdot \overline{14}$ \\
\hline Immediate oocyst rate $(R)$ & $4 \cdot 3$ & $4 \cdot 5$ & $4 \cdot 5$ & $5 \cdot 0$ \\
\hline Infectious reservoir $(K)$ & 4.9 & $4 \cdot 2$ & $3 \cdot 6$ & $2 \cdot 8$ \\
\hline Survival per gonotrophic cycle $(P)$ & 50 & $52 \cdot 9$ & 58 & $65 \cdot 5$ \\
\hline
\end{tabular}

${ }^{a}$ The estimated and expected proportions of the mixed infections (i.e., infections acquired at both the last and previous feeds) determined by the mixture decomposition method are constrained to be the same.

from the most recent feed. This allowed us to classify each oocyst separately and hence to classify mosquitoes according to whether they had acquired an infection at only the latest, at only the previous, or at both the latest and previous feeds (Table). If the probability that a mosquito will become infected at any given feed $(K)$ is independent of the probability that it is already infected $(R)$, then the expected number of mosquitoes with mixed infections in this sense is $R K$. In both species, this expected number of mixed infections was much less than the number of mixed infections estimated by using the cut-off size of $17 \cdot 5 \mu \mathrm{m}$ (Table).

The second approach was to use the mixture decomposition method to resolve the overall oocyst size distributions into 2 components corresponding to $\theta(x)$ and $\phi(x)$. This analysis indicated that the proportion of oocysts in $A$. funestus derived from previous feed(s) $(\lambda)$ was 0.622 (SE $=0.044)$. In those $A$. funestus which carried infections derived from both the last feed and previous feed(s), the proportion of oocysts from the previous feed(s) $(\beta)$ was estimated to be $0-878$. The equivalent estimates for $A$. gambiae s.l. were $\lambda=0.860(\mathrm{sE}=0.044)$ and $\beta=0.773$. Corresponding to this, the proportions of oocysts attributed to the most recent feeds $(1-\lambda)$ were much less than the percentages of infected mosquitoes thought to have gained an infection at that feed $(48.8 \%$ of infected $A$. funestus and $36.4 \%$ of infected $A$. gambiae s.l.). The overlap in distributions was particularly noticeable for $A$. gambiae s.l. Careful examination of the Figure suggests that the overlap was due mainly to oocysts from previous feeds which had grown slowly. Corresponding oocysts from the latest feed would not be detectable at 3 d. This could explain the large discrepancy between the total numbers of oocysts attributed to the latest and to previous feeds.

Estimates of $K$ (the infectious reservoir) and of the survival rate, based on these 2 methods of analysis, are shown in the Table.

\section{Discussion}

Measurements of oocyst sizes in wild-caught mosquitoes after blood meal digestion provide a simple way of obtaining estimates of mosquito survival and of the size of the infectious reservoir.

The method has not been applied previously, probably because of concern that oocyst growth rates are variable (SHUTE \& MARYON, 1952), leading to the misclassification of many infections if a single cut-off value is used to discriminate between them. The laboratory controls presumably indicated correctly the extent of variability in uocyst size under optimal conditions and with genetically homogenous parasites. In a natural population of parasites one would expect oocyst growth rates to be more heterogenous, because of variability in the conditions experienced by the mosquitoes and ge- netic variation of the parasites, even within one mosquito (BABIKER et al., 1994).

Misclassification of vocysts can lead to overestimation of either $K$ or $R$. If the oocysts are classified using a single cut-off value chosen to assign correctly as many laboratory-derived oocysts as possible, then the number of mosquitoes with mixed infections is also overestimated, resulting in overestimates of both $K$ and $R$ (and $P$ ). For example, our cut-off size of $17.5 \mu \mathrm{m}$ would classity an insect with 5 oocysts, 4 of which were $22 \mu \mathrm{m}$ in diameter and one of which was $17 \mu \mathrm{m}$, as containing a mixed infection. Such a mosquito has, however, probably become infected only once. Such misclassification seems likely to account for the excess in the numbers of mixed infections (although there are several other possibilities, such as that some mosquitoes preferentially feed on infected hosts).

Our proposed statistical model addresses overestimation of mixed infections. This model determines the extent of misclassification and allows for it in the estimation of $K$ and $R$, assuming independence of infections. This analysis suggested that the cut-off value of $17.5 \mu \mathrm{m}$ wrongly allocated $39.8 \%$ of oocysts in $A$. gambiae s.l. and $18.3 \%$ in $A$. funestus, and that more of the oocysts observed were derived from previous feeds than use of the cut-off diameter suggested.

The statistical model also gave lower estimates of $K$ and of $R$ than did the cut-off diameter, but the difference between the methods was not very large. This implies that the use of a single cut-off value to classify oocysts gives estimates of infectiousness and of mosquito survival that could serve as acceptable first approximations for many purposes. Nevertheless, the most reliable estimates of $K$ for the neighbouring village of Namawala were smaller than any found in the present study (J. D. Charlwood et al., in preparation). This suggests that the estimates from the statistical model, which were lower, are probably more reliable. The estimated survival per gonotrophic cycle was similar using the 2 different methods, and comparable to determinations made from parous rates in Namawala (J. D. Charlwood et al., in preparation ) $(61 \cdot 1 \%$ for $A$. funestus and $62.3 \%$ for $A$. gam biae s.l.). The higher survival estimates (those from the statistical model) were more consistent with those from other techniques.

A reason for care in applying the new approach is that the microscopical detection of young oocysts with a dry $\times 40$ objective is likely to be much less sensitive than that of older oocysts. In both species of mosquitoes, the percentage of oocysts attributed to the most recent feeds was much less than the percentage of mosquitoes thought to have gained an infection at that feed. These differences cannot be entirely explained by a small proportion of mosquitoes with a high multiplicity of infections, but the presence of slowly growing oocysts that 
had not yet become visible could provide an explanation. These small oocysts are presumably less likely to mature before the insect dies, and are therefore of lesser epidemiological significance. However, further studies are needed to determine the distributions of oocyst sizes in naturally infected mosquitoes over the whole of the extrinsic cycle.

Because the control oocysts were obtained by a technique that was less than optimal, it would be useful to evaluate the method using alternative controls. Improved controls with which to estimate oocyst sizes from the previous feed, $\theta(x)$, might be either examination of one-parous mosquitoes killed on arriving for their second feed or determination of oocyst sizes in 'wild' mosquitoes raised from pupae, allowed to feed on infected hosts, and then kept for one gonotrophic cycle. As a simpler alternative, $\phi(x)$ could be determined from the oocyst sizes in wild-caught mosquitoes that had fed on a known non-infective host and again been maintained in captivity for the duration of one cycle.

The identification of oocysts derived from the most recent feed by measuring their diameters is a simple modification to procedures for determining the infection status of mosquitoes. If the mebromine staining technique is used, the mosquitoes need be kept only until the blood meal has been digested. The preservation of mosquito midguts also allows for batch processing of material. All these considerations make the technique practicable for large-scale field studies of the transmission of malaria from humans to mosquitoes.

\section{Acknowledgements}

We thank the villagers of Kisegese for their participation in this study, the field assistants, Anniseth Kihonda, Kanisius Mscrengc and Romanus Ndunguru, for collecting and dissceting the mosquitoes, and Penelope Vounatsou for statistical assistance. We received generous help from $T$. Lensen, $M$. Bolmer and G. J. Van Gemert for production of oocysts, Prof Marcel Tanner and Prof. Wen Kilama (Director General, National Institute of Medical Research, Tanzania). Funds for this project were provided by the Director General of Development Co-operation (DGIS NL 002701) of the Dutch Government and the Rudolf Geigy Foundation. The study was also partially supported by Swiss National Science Foundation Grant no. 3243527.95. Research clearance was granted by the Tanzania Commission for Science and Technology, reference NSR/RCA 90.

\section{References}

Babiker, H. A., Ranford-Cartwright, L. C., Currie, D., Charlwood, D., Billingsley, P., Teuscher, R. \& Walliker, D. (1994). Random mating in a natural population of the malaria parasite Plasmodium falciparum. Parasitology, 109, 413-421.

Babiker, H. A., Charlwood, J. D., Smith, T. \& Walker, D.

(1995). Gene flow and cross-mating in Plasmodium falciparum in households in a Tanzanian village. Parasitology, 111, 433-442.

Billingsley, P. F., Medley, G. F., Charlwood, J. D. \& Sinden, R. E. (1994). Relationship between prevalence and intensity of Plasmodium falciparum infection in natural populations of Anopheles mosquitoes. American Fournal of Tropical Medicine and Hygiene, 51, 260-270.

Charlwood, J. D., Kihonda, J., Sama, S., Billingsley, P. F. Hadji, H., Verhave, J. P., Lyimo, E. \& Smith, T. (1995). The rise and fall of $A$ nopheles gambiae in a Tanzanian villagc. $B u$ letin of Entomological Research, 85, 37-44.

Draper, C. C. (1953). Observations on the infectiousness of gametocytes in hyperendemic malaria. Transactions of the Royal Society of Tropical Medicine and Hygiene, 47, 160-165.

Githeko, A. K., Brandling-Bennett, A. D., Beier, M., Atieli, F. Owaga, M. \& Collins, F. H. (1992). The reservoir of Plasmodium falciparum malaria in a holoendemic area of western Kenya. Transactions of the Royal Society of Tropical Medicine and Hygiene, 86, 355-358.

Graves, P. M., Burkot, T. R., Carter, R., Cattani, J. A., Lagog, M., Parker, J., Brabin, B. J., Gibson, F. D., Bradley, D. J. \& Alpers, M.P. (1988). Measurement of malaria infectivity of human populations to mosquitoes in the Madang area, Papua New Guinea. Parasitology, 97, 251-263.

Graves, P. M., Burkot, T. R., Saul, A. J., Hayes, R. J. \& Carter,
R. (1990). Estimation of anopheline survival rate, vectorial capacity and mosquito infection probability from malaria vector infection rates in villages near Madang, Papua New Guinea. Fournal of Applied Ecology, 27, 134-147.

Haji, H., Smith, T., Charlwood, J. D. \& Meuwissen, J. Th. (in press). Absence of relationships between selected host factors and natural infectivity of Plasmodium falciparum in an area of high transmission. Parasitology.

Muirhead-Thomson, R. C. (1954). Factors determining the true reservoir of Plasmodium falciparum and Wuchereria bancrofit in a West African Village. Transactions of the Royal Society of Tropical Medicine and Hygiene, 48, 208-225.

Muirhead-Thomson, R. C. (1957). Notes on the characters of Plasmodium malariae oocysts of possible value in mixed infections. American Fournal of Tropical Medicine and Hygiene, 6 980-985.

Muirhead-Thomson, R.C. \& Mercier, E. C. (1952). Factors in malaria transmission in Anopheles albimanus in Jamaica. An nals of Tropical Medicine and Parasitology, 46, 103-116.

Service, M. W. (1993). Mosquito Ecology. Field Sampling Methods. London \& New York: Elsevier Applied Science.

Shute, P. G. \& Maryon, M. (1952). A study on human malaria oocysts as an aid to species diagnosis. Transactions of the Royal Society of Tropical Medicine and Hygiene, 46, 275-292

Thuikam, T., Mulder, B., Dechering, K., Stoffels, H., Verhave, J.-P., Cot, M., Carnavale, P., Meuwissen, J. E. Th. \& Robert, V. (1993). Experimental infections of Anopheles gambiae with Plasmodium falciparum of naturally infected gametocyte carriers in Cameroon: factors influencing the infectivity to mosquitoes. Tropical Medicine and Parasitology, 44, 271-176.

Vounatsou, P., Smith, T. \& Smith, A. F. M. (in press). Bayesian analysis of two component mixture distributions: application to the estimation of malaria attributable fractions. Applied Statistics.

Received 5 February 1996; accepted for publication 18 April 1996

\section{Appendix}

Estimation of infection rates allowing for poor resolution of oocyst sizes

In order to compute the likelihood for $K$ (or, equivalently, $R$ ), the likelihood of the observed vector of oocyst sizes $x_{j}=\left(x_{1, j}, x_{2, j}, x_{3, j}, \ldots x_{n, j}\right)$ for each mosquito $j$, with $n$ oocysts, must be computed. Conditional on mosquito $j$ having acquircd infections only at the last fecd, this likelihood is

$L\left(\underline{x}_{j}\right.$ infected at latest feed only $)=\prod_{i=1}^{n} \theta\left(x_{i, j}\right)$

Conditional on mosquito $j$ having acquired infections only at previous feed(s), the likelihood is

$L\left(\underline{x}_{j}\right.$ infected at previous feed(s) only $)=\prod_{i=1}^{n} \phi\left(x_{i, j}\right)$

The oocyst sizes in mosquitoes with infections acquired from both the last and previous feed(s) are a sample from

$$
f^{\prime}(x) \sim(1-\beta) \theta(x)+\beta \phi(x)
$$

and the contribution to the likelihood for such mosquitoes is then

$L\left(\underline{x}_{j}\right.$ infected both latest and previous feeds $)=$

$$
\prod_{i=1}^{n}\left((1-\beta) \theta\left(x_{i, j}\right)+\beta \phi\left(x_{i, j}\right)\right)
$$

it follows that the unconditional likelihood for mosquito $j$ is

$L\left(x_{j}=K^{\prime}(1-R) \Pi \theta\left(x_{i, j}\right)+R(1-K) \Pi \phi\left(x_{i, j}\right)+R K \Pi\left((1-\beta) \theta\left(x_{i, j}\right)+\beta \phi\left(x_{i, j}\right)\right)\right.$.

By maximizing the product of these likelihoods for the whole population of infected mosquitoes, with respect to $K$ (or, equivalently, $R$ ), an estimate of the probability of infection per feed can be obtained. 